\title{
Impacts of Air-Sea Interaction on Tropical Cyclone Track and Intensity
}

\author{
LigUANG WU \\ Goddard Earth and Technology Center, University of Maryland, Baltimore County, Baltimore, and Laboratory for Atmospheres, \\ NASA Goddard Space Flight Center, Greenbelt, Maryland \\ BIN WANG \\ Department of Meteorology, School of Ocean and Earth Science and Technology, University of Hawaii at Manoa, Honolulu, Hawaii \\ SCOTT A. BRAUN \\ Laboratory for Atmospheres, NASA Goddard Space Flight Center, Greenbelt, Maryland
}

(Manuscript received 15 July 2004, in final form 19 April 2005)

\begin{abstract}
While the previous studies of the impacts of air-sea interaction on tropical cyclones (TCs) generally agree on significant reduction in intensity and little change in track, they did not further explore the relative roles of the weak symmetric and strong asymmetric sea surface temperature (SST) anomalies relative to the TC center. These issues are investigated numerically with a coupled hurricane-ocean model in this study.

Despite the relatively small magnitude compared to the asymmetric component of the resulting cooling, the symmetric cooling plays a decisive role in weakening TC intensity. A likely reason is that the symmetric cooling directly reduces the TC intensity, while the asymmetric cooling affects the intensity through the resulting TC asymmetries, which are mainly confined to the lower boundary and much weaker than those resulting from large-scale environmental influences.

The differences in TC tracks between the coupled and fixed SST experiments are generally small because of the competing processes associated with the changes in TC asymmetries and the beta drift induced by air-sea interaction. The symmetric component of the SST drop weakens the TC intensity and outer strength, leading to a more northward beta drift. On the other hand, since the asymmetric component of the SST cooling is negative in the rear and positive in the front of a TC in the coupled experiments, the enhanced diabatic heating is on the southern side of a westward-moving TC, tending to shift the TC southward. In the coupled model the westward TCs with relatively weak (strong) outer strength tend to turn to the north (south) of the corresponding TCs without air-sea interaction.
\end{abstract}

\section{Introduction}

A tropical cyclone (TC) develops and is maintained by drawing energy from the underlying ocean surface. It can form only over waters of $26^{\circ} \mathrm{C}$ or higher and its intensity is very sensitive to the sea surface temperature (SST; e.g., Tuleya and Kurihara 1982; Emanuel 1986). Treating a tropical storm as a Carnot heat engine, Emanuel (1986) suggested that the TC maximum po-

Corresponding author address: Dr. Liguang Wu, NASA GSFC, Code 912, Greenbelt, MD 20771.

E-mail: liguang@agnes.gsfc.nasa.gov tential intensity is primarily determined by the underlying SST. At the same time, the surface wind stress associated with a TC can generate strong turbulent mixing that deepens the ocean mixed layer (OML) by entraining cooler water into the surface layer, leading to significant SST decreases. Observations indicate that the SST cooling caused by TCs ranges from $1^{\circ}$ to $6^{\circ} \mathrm{C}$ (Price 1981).

The feedback of the resulting cooling on TC intensity has been investigated using coupled hurricane-ocean models. Early experiments were performed with upper OML models forced by axisymmetric TC models (Elsberry et al. 1976; Chang and Anthes 1979; Sutyrin and Khain 1979). Because of the markedly rightward bias of 
the ocean response with respect to the TC track, threedimensional coupled models were used recently (Bender et al. 1993; Falkovich et al. 1995; Chan et al. 2001; Bender and Ginis 2000). These simulations indicated that the storm-induced cooling of the OML can significantly weaken a storm. However, given the fact that the resulting SST anomalies comprise the axially symmetric and asymmetric components relative to the TC center, the relative contribution of each component to TC motion and track has not addressed. Emanuel's (1999) study suggests that the symmetric component of the storm-induced SST anomaly field may play a decisive role in reducing the storm intensity because he was able to successfully simulate most aspects of the evolution of storm intensity using a two-dimensional (axially symmetric) hurricane model coupled with a onedimensional ocean model oriented along the storm track. Whether the symmetric SST anomalies can be fully responsible for the reduction of storm intensity will be examined here.

While the previous studies generally agree regarding the reduction in TC intensity, they disagree concerning the impacts of air-sea interaction on TC motion. Khain and Ginis (1991) found that westward-moving (eastward moving) TCs in coupled experiments were displaced to the south (north) relative to the corresponding experiments without air-sea interaction. They attributed these track differences to asymmetric precipitation patterns that were shifted azimuthally because of air-sea interaction. On the other hand, the Bender et al. (1993) experiments with the National Oceanic and Atmospheric Administration (NOAA) Geophysical Fluid Dynamics Laboratory (GFDL) TC model coupled with an eight-layer ocean model found that the westward tracks gradually turned more to the north relative to the track in the fixed SST experiments, especially for slow-moving storms. Bender et al. (1993) suggested that this track deviation is related to a systematic decrease in the azimuthally averaged tangential flow of the TC vortex. In general, the influence of airsea on TC tracks is small but persistent. This study will make an effort to understand what causes these contradictory results.

This study particularly focuses on the different ways the symmetric and asymmetric components of the storm-induced SST anomaly affect TC intensity and motion. Although high-resolution models with more sophisticated model physics can simulate the very details of real hurricanes, a coupled hurricane-ocean model that can capture the primarily features of hurricane-ocean interaction but with intermediate complexity will be used in the present study.

\section{The coupled hurricane-ocean model and experimental design}

\section{a. Hurricane model}

The hurricane model designed by Wang (1998) consists of $201 \times 201$ grid points with a uniform spacing of $25 \mathrm{~km}$ in the horizontal and 16 vertical layers with relatively high resolution near the lower and upper boundaries. The details of the model and its capability to simulate the motion and evolution of baroclinic TCs in the presence of diabatic heating has been documented by $\mathrm{Wu}(1999)$ and $\mathrm{Wu}$ and Wang (2000). The primary model physics include large-scale condensation calculated explicitly with the method used by Leslie et al. (1985), subgrid-scale cumulus convection parameterized with Kuo's (1974) scheme, a Newtonian cooling as used in the TC model by Rotunno and Emanuel (1987), and surface fluxes of momentum, sensible and latent heat calculated by the bulk aerodynamic method, in which the exchange coefficients are determined following Kondo (1975) and Louis (1979).

\section{b. Ocean model}

The ocean response to the forcing of a moving hurricane may be conveniently divided into the forced and relaxation stages (Gill 1984). The forced stage response which typically lasts half a day or a storm residence time, is a primarily local response and includes OML currents of $1 \mathrm{~m} \mathrm{~s}^{-1}$ and substantial cooling of the OML primarily in the right rear quadrant by the vertical mixing (Price 1981; Sanford et al. 1987; Black 1983; Shay et al. 1992). The relaxation stage response following a hurricane passage is an inherently nonlocal baroclinic response to the wind stress curl and typically lasts 5-10 days. The energy is spread through internal waves (Geisler 1970; Gill 1984) that penetrate into the thermocline (Shay and Elsberry 1987; Brink 1989), and leaves behind a baroclinic geostrophic current along the storm track. To simulate these ocean responses, the ocean model should include OML physics and thermocline and upper ocean dynamics.

A two-and-a-half layer ocean model developed by Wang et al. (1995) is used in this study. Since the response is primarily baroclinic, the ocean upper boundary is a rigid lid so that the barotropic response is removed. The model includes two active upper layers: an OML and a middle thermocline layer. Below the thermocline layer is a motionless deep layer $(z<-h)$ in which the temperature $\left(T_{r}\right)$ is assumed to be constant. In the OML $\left(0>z>-h_{1}\right)$, the temperature $\left(T_{1}\right)$ and velocity are independent of depth, and in the thermocline layer $\left(-h_{1}>z>-h\right)$, the temperature decreases linearly from $T_{1}$ to $T_{r}$. 
TABLE 1. Summary of the numerical experiments.

\begin{tabular}{lcccc}
\hline \hline & Fixed SST & Coupled & Symmetric SST forcing & Asymmetric SST forcing \\
\hline E1: $f$ plane, $-4 \mathrm{~m} \mathrm{~s}^{-1}$ & E1F & E1C & E1S & E1A \\
E2: $\beta$ plane, $0 \mathrm{~m} \mathrm{~s}^{-1}$ & E2F & E2C & E2S & E2A \\
E3: $\beta$ plane, $-4 \mathrm{~m} \mathrm{~s}^{-1}$ & E3F & E3C & E3S & E3A \\
\hline
\end{tabular}

Since the ocean model was originally developed for study of ocean phenomena on the interannual time scale, a number of aspects of the model physics are modified to better simulate the ocean response to the forcing of a moving hurricane. First, following Bender et al. (1993), the Kraus-Turner scheme (Kraus and Turner 1967) to parameterize the vertical turbulent mixing (entrainment) is replaced by the Deardorff's scheme (Deardorff 1983) to include the important shear instability (Ginis 1995). According to Deardorff (1983), a singularity occurs in the Kraus-Turner scheme when the velocity shear is significant. Second, in the modified model it is assumed that the downward heat flux decreases exponentially in the mixed layer and the turbulent momentum and heat fluxes are not allowed to penetrate below the OML base. The TCocean interaction is through the surface turbulent fluxes of momentum, sensible and latent heat. The energetics of the OML has been studied extensively for the last two decades. Although no consensus exists so far on the amount of turbulent energy radiated from the mixed layer, some theoretical and laboratory analyses suggest that the bulk of the energy fed into the mixed layer is trapped by the transition layer and is eventually dissipated by wave breaking (Fernando 1991). This scenario is probably appropriate for the ocean areas that experience the most intense TCs (Ginis 1995). Third, parameterizations of the temperature of entrained water are a key to closure of the OML equations (Wang et al. 1995). The original model considers a thin entrainment layer existing just below the OML base. This entrainment layer is a thin region of vigorous small-scale mixing, within which the turbulent flux drops sharply from a finite value to zero. The vertical temperature gradient in the entrainment layer with thickness $\Delta h_{e}$ is assumed to be proportional to the mean vertical temperature gradient in the thermocline layer, that is,

$$
T_{1}-T_{e}=k \Delta h_{e} \frac{T_{1}-T_{r}}{h-h_{1}},
$$

where $T_{e}$ is the temperature of the entrainment layer and $k=1$ was used in the original model. In the response of ocean to the TC forcing shown by Shay et al. (1992), the temperature gradient between the OML and the top of the thermocline can be much larger than the mean vertical temperature gradient in the thermocline. Based on the results of Shay et al. (1992), we assume $k=3$.

The ocean is initially assumed to be horizontally homogeneous and quiescent. The water temperature in the deep resting layer is set to $10^{\circ} \mathrm{C}$. The entrainment layer depth is $5 \mathrm{~m}$, which is identical to that used by Wang et al. (1995). The initial OML depth and temperature are also two important parameters. Sensitivity tests show that the magnitude of the cooling in this layer increases with decreasing initial depth or increasing initial SST. Moreover, the magnitude of the OML cooling also increases with increasing depth or temperature gradient associated with the thin entrainment layer. However, the cooling patterns are generally similar. For this reason, the depth of the mixed layer is initially set to $35 \mathrm{~m}$ in all coupled experiments. The ocean model has the same grid spacing and domain size as the hurricane model. The SST and wind stress are passed between the hurricane and ocean models every $3 \mathrm{~min}$. Periodic lateral boundary conditions are used in the hurricane and ocean models.

\section{c. Experimental design}

Three sets of idealized numerical experiments are conducted with different environmental influences (Table 1). The ocean response depends on whether the TC speed exceeds the long gravity wave speed or not. In the current ocean model, the phase velocity of long gravity waves is about $1.2 \mathrm{~m} \mathrm{~s}^{-1}$ for the first baroclinic mode. The simulation of the cold wake behind a storm requires that the translation speed of the $\mathrm{TC}$ is greater than the gravity wave speed. The first set of numerical experiments (E1) are designed to be the simplest case, which are run on an $\mathrm{f}$ plane with a horizontally uniform easterly ambient flow $-4 \mathrm{~m} \mathrm{~s}^{-1}$. The second set of numerical experiments (E2) are on a beta plane in a resting environment, in which the vortex movement arises from the beta drift. The third set of numerical experiments (E3) are a combination of E1 and E2, that is, they include the influences of uniform easterly flow and the beta effect.

For each set, four experiments are conducted: fixed SST, coupled, symmetric-only SST forcing, and asym- 
metric-only SST forcing (Table 1). For example, E1F, $\mathrm{E} 1 \mathrm{C}, \mathrm{E} 1 \mathrm{~S}$, and E1A, respectively, are referred to these four experiments in set E1. In the fixed SST experiments, the air-sea coupling is excluded and the SSTs are fixed in time and horizontally uniform $\left(29.5^{\circ} \mathrm{C}\right.$ for $\mathrm{E} 1$ and $28.5^{\circ} \mathrm{C}$ for $\mathrm{E} 2$ and E3). The coupled experiments are run with full air-sea coupling. The SST anomalies in the coupled experiments are saved each hour and decomposed into their symmetric and asymmetric components with respect to the TC center. The symmetric SST component can directly affect the intensity of the TC's symmetric circulation while the asymmetric component affects the TC through the interaction between the mean TC circulation and the induced asymmetric circulation. Because the different ways the two components influence TC intensity and track are different, two more uncoupled experiments are run using the sum of the horizontal uniform SST and the timedependent symmetric or asymmetric SST anomalies derived from the corresponding coupled experiments as the SST forcing, respectively. For convenience, these experiments are called symmetric-only and asymmetric-only SST forcing experiments in this paper.

All experiments begin with an identical, initially symmetric baroclinic vortex. The intensity of the initial vortex decreases with height, but without an anticyclonic circulation atop. The maximum tangential wind $\left(v_{m}\right)$ of $25 \mathrm{~m} \mathrm{~s}^{-1}$ at $r_{m}=100 \mathrm{~km}$ is at the lowest model level. The horizontal wind profile $[v(r)]$ is generated following

$$
v(r)=v_{m}\left(\frac{r}{r_{m}}\right) \exp \frac{1}{b}\left[1-\left(\frac{r}{r_{m}}\right)^{b}\right],
$$

where $r$ and $b$ are the radius from the TC center and the shape parameter, respectively. Here, $b$ is set to 0.5 in sets E1, E2, and E3. The change in this parameter primarily affects the outer strength of the initial vortex, which affects the magnitude of the beta drift. To examine the influence of air-sea interaction on the beta drift, three additional experiments conducted are the same as E2 except that the shape parameter is 0.3 for B1, 1.0 for $\mathrm{B} 2$, and 1.2 for $\mathrm{B} 3$.

\section{Ocean response in the coupled model}

In this section we focus on the coupled experiment in set E2 (E2C) since the general patterns of the ocean response to TC forcing are in general similar for all the experiments. The time series of the maximum current speeds in the OML and thermocline layer and the minimum OML temperature are shown in Figs. 1a,b. The OML current increases quickly while the minimum
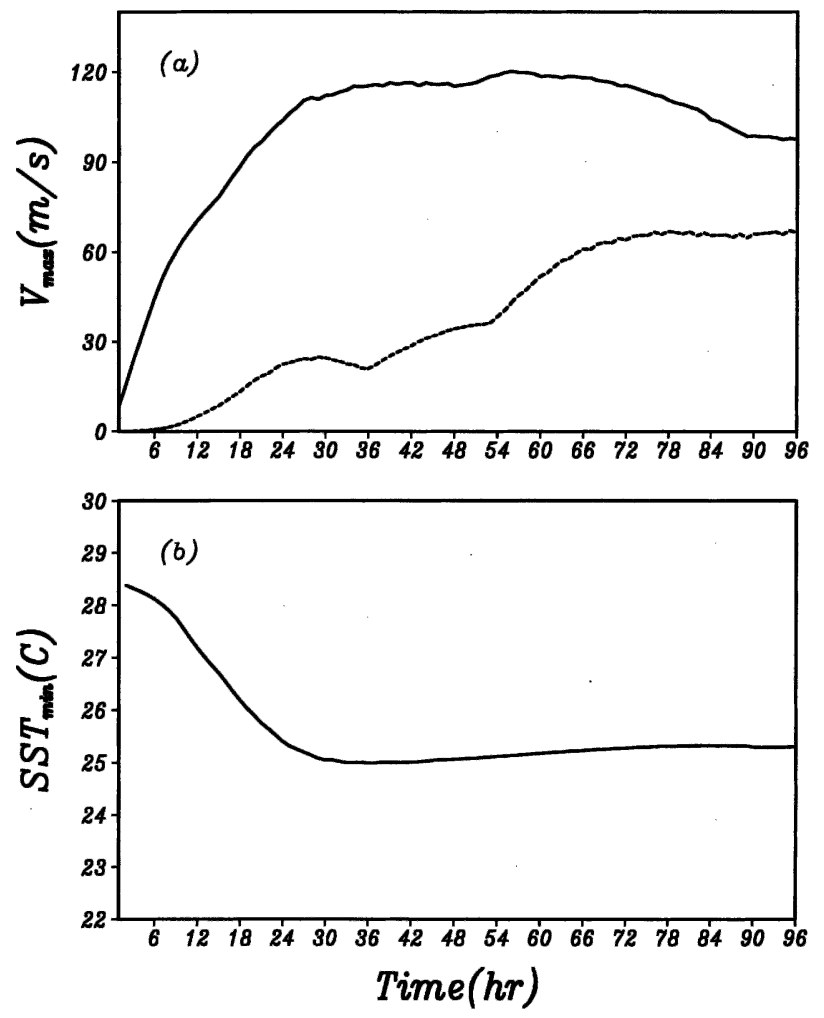

FIG. 1. Time series of the maximum ocean responses: (a) currents $\left(\mathrm{cm} \mathrm{s}^{-1}\right)$ in the mixed (solid) and thermocline (dashed) layers, and (b) sea surface temperature.

temperature decreases rapidly during the first $24 \mathrm{~h}$, and both reach a quasi-steady state after $24 \mathrm{~h}$. However, the thermocline current continues to accelerate until $72 \mathrm{~h}$. The maximum mixed layer cooling of $3.5^{\circ} \mathrm{C}$ at $33 \mathrm{~h}$ is generally consistent with ocean observations since the storm translation due to the beta drift is only $2-4 \mathrm{~m} \mathrm{~s}^{-1}$. Black (1983) found that storms moving faster (slower) than $3 \mathrm{~m} \mathrm{~s}^{-1}$ can produce $1^{\circ}-3^{\circ} \mathrm{C}\left(3^{\circ}-5^{\circ} \mathrm{C}\right)$ cooling. When the SST reaches its minimum value, the OML current is about $1.1 \mathrm{~m} \mathrm{~s}^{-1}$. Thereafter, the current increases slowly and attains its maximum of $1.2 \mathrm{~m} \mathrm{~s}^{-1}$ at $56 \mathrm{~h}$. The magnitude of the resulting OML currents also compares favorably with observations (e.g., Shay et al. 1992) and numerical simulations with other sophisticated coupled models (e.g., Bender et al. 1993).

The ocean responses at $96 \mathrm{~h}$ in E2C have negative SST anomalies over a large area with a cold wake along the TC track (Fig. 2a). The cooling has a pronounced rightward bias with respect to the TC track and the maximum cooling is immediately behind the TC center. The pattern of the OML cooling is consistent with the rightward-biased OML deepening (Fig. 2b). The largest entrainment rate, which is parameterized as a function of wind stress, velocity shear at the base of the mixed 

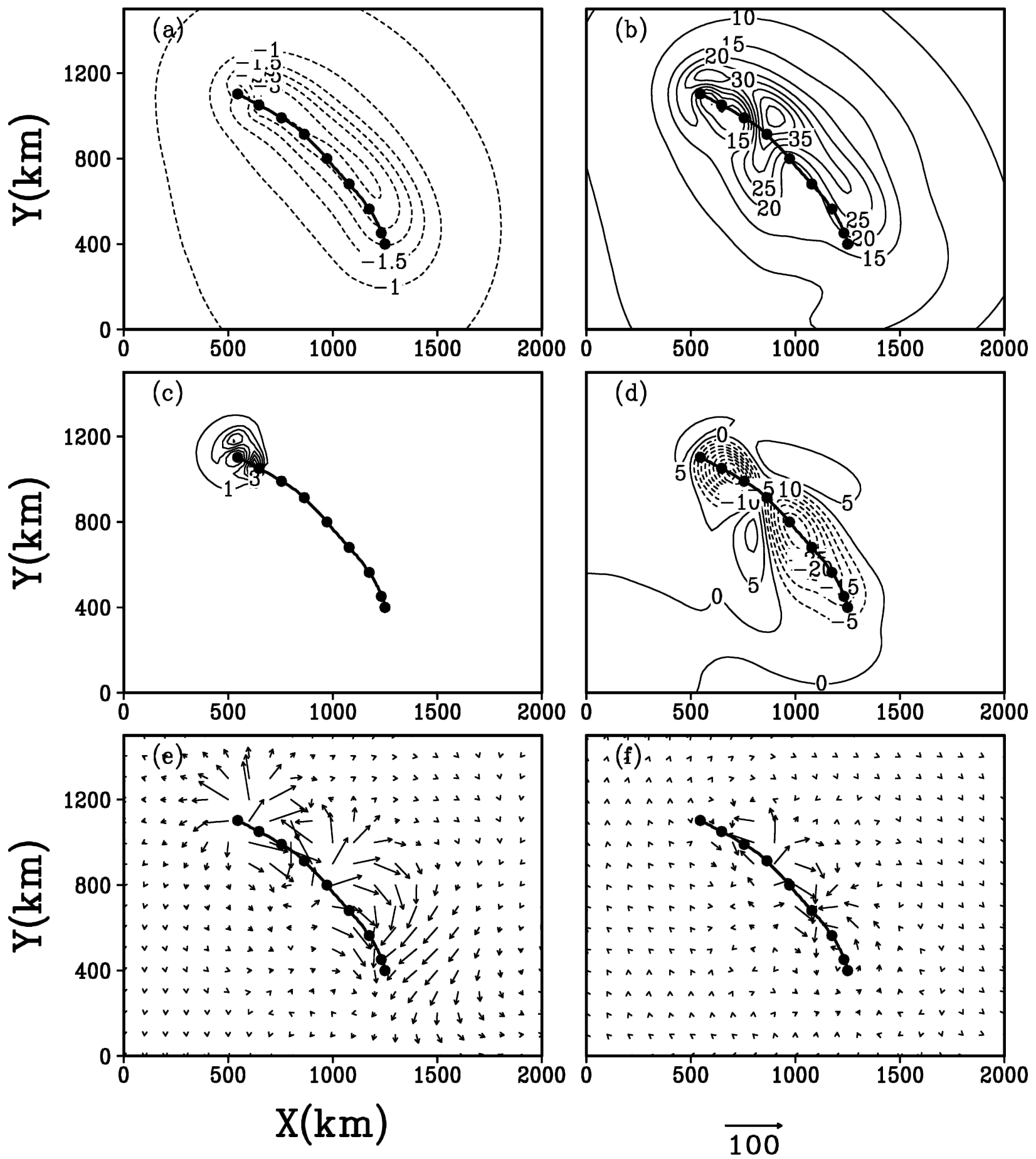

FIG. 2. Ocean response by $96 \mathrm{~h}$ in the coupled experiment E2C: (a) sea surface temperature anomaly $\left({ }^{\circ} \mathrm{C}\right.$ ); (b) mixed layer depth anomaly (m, positive values indicate greater depths); (c) entrainment rate $\left(\mathrm{cm} \mathrm{s}^{-1}\right)$; (d) thermocline depth anomaly ( $\mathrm{m}$, negative values indicate shallowing depths); (e) currents in the mixed layer $\left(\mathrm{cm} \mathrm{s}^{-1}\right)$, and (f) currents in the thermocline layer $\left(\mathrm{cm} \mathrm{s}^{-1}\right)$. The TC track starts from the rhs and the center positions every $12 \mathrm{~h}$ are indicated with closed circles.

layer, and convective overturning due to the surface buoyancy fluxes, is primarily confined to the vicinity of the TC center (Fig. 2c). However, the ocean inertiagravity waves excited by moving TCs also contribute some OML deepening in the wake of the TC (Gill 1984).

The region of the maximum OML deepening occurs well behind the TC center on the right side of the TC 
track. The maximum deepening reaches $63 \mathrm{~m}$ at $44 \mathrm{~h}$ and decreases to about $45 \mathrm{~m}$ at $96 \mathrm{~h}$. This is also comparable with the observational analysis of Hurricane Gilbert (1988) by Shay et al. (1992). They found that storm passage increased the OML depth from prestorm values of $30-35 \mathrm{~m}$ to approximately $60 \mathrm{~m}$ on the righthand side of the track. The pattern of OML deepening is also similar to that simulated with the GFDL coupled model for Hurricane Norbert (Bender et al. 1993). The model OML currents are presented in Fig. 2e. In the front of the TC, the cyclonically rotating wind stress generates OML currents with significant divergence and the current pattern becomes significantly asymmetric behind the TC. The thermocline depth has negative anomalies behind the TC center without significant biases with respect to the track (Fig. 2d). The slightly positive anomalies outside the negative anomalies are an indication of the horizontal dispersion of inertiagravity waves after the TC passing. Compared to the OML currents, the thermocline currents, which are primarily driven by the depth gradient (Bender et al. 1993), are relatively small (Fig. 2f).

In summary, the major features of the simulated ocean response in the current coupled hurricane-ocean model, that is, the amplitudes and spatial patterns of the OML cooling and deepening, the thermocline depth anomalies, and the induced ocean currents in the mixed and thermocline layers, are all in good agreement with observations and previous numerical simulations. Therefore, the current ocean model is capable of realistically simulating the response of the ocean to slowly moving TCs as in this experiment.

\section{Influence of air-sea interaction on TCs}

\section{a. Intensity change}

The TC intensities for sets E1, E2, and E3 are compared with the corresponding fixed-SST experiments in terms of the maximum wind speed and the minimum central pressure (Fig. 3). In agreement with previous numerical studies (e.g., Chang and Anthes 1979; Khain and Ginis 1991; Bender et al. 1993; Bender and Ginis 2000), the present simulations confirm that air-sea interaction reduces TC intensity as a result of mixed layer cooling. In set E1, the TC reaches a maximum wind of $60.1 \mathrm{~m} \mathrm{~s}^{-1}$ and a central pressure of $917 \mathrm{mb}$ without the inclusion of air-sea interaction (E1F). When the airsea coupling is included (E1C), the maximum wind is reduced by about $12 \mathrm{~m} \mathrm{~s}^{-1}$ and the central surface pressure decreases by $32 \mathrm{mb}$. In set E2, the coupling leads to differences of $14 \mathrm{~m} \mathrm{~s}^{-1}$ in the maximum wind and $24 \mathrm{mb}$ in the minimum central pressure. When the beta effect and environmental flow are combined (E3), the air-sea interaction reduces the maximum wind by $10 \mathrm{~m} \mathrm{~s}^{-1}$ and increases the central pressure by $18 \mathrm{mb}$.

The decomposition of the SST anomaly field shown in Fig. 2a shows that the symmetric SST component extends over a large area with an average magnitude of $1.5^{\circ} \mathrm{C}$ in the inner core region (Fig. 4a) and that the asymmetric SST pattern has a cold wake of negative anomalies behind the center and positive anomalies in front of the center (Fig. 4b). As shown in Fig. 3, the TC intensities in the symmetric-only and asymmetric-only SST forcing experiments are nearly identical to those in the coupled and fixed-SST experiments, respectively, except for small fluctuations. This result suggests that the intensity reduction in the coupled experiments is primarily caused by the symmetric component of the induced SST drop, while the asymmetric component of the SST anomalies resulting from air-sea coupling only plays a minor role in the intensity change.

In principle, the asymmetric SST field can affect TC intensity only through the resulting inner-core TC asymmetries. To examine the asymmetries associated with the asymmetric SST forcing, the asymmetric winds in the fixed-SST experiments are compared to those in the asymmetric-only SST forcing experiments. The differences of the asymmetric winds between these two experiments are considered to be the primary result of the asymmetric SST forcing. Compared to the asymmetric winds resulting from the beta effect (Fig. 5a), the winds induced by the asymmetric SST forcing (Fig. 5b) are mainly confined to a small region associated with the significant SST anomalies, with the largest differences over the warm anomaly. Moreover, the resulting asymmetric winds are confined primarily to the lower model levels (figure not shown). Therefore the asymmetries induced by the asymmetric SST forcing play an insignificant role in TC intensity change.

\section{b. The TC tracks}

The TC tracks between the coupled and fixed SST experiments are shown in Fig. 6. The influence of airsea coupling on TC tracks is relatively small but persistent. In agreement with those of the westward-moving TCs simulated by Khain and Ginis (1991), the TCs in the coupled experiments are displaced to the south relative to the corresponding experiments without airsea interaction for sets E1, E2, and E3. By $96 \mathrm{~h}$, the track differences for sets E1, E2, and E3 are 100, 29, and $40 \mathrm{~km}$, respectively.

The asymmetric convection with respect to a TC center can directly affect TC motion. Wu and Wang (2001) suggested that wavenumber-1 diabatic heating with respect to the TC center can generate a positive potential vorticity tendency and thus can shift the TC to the 

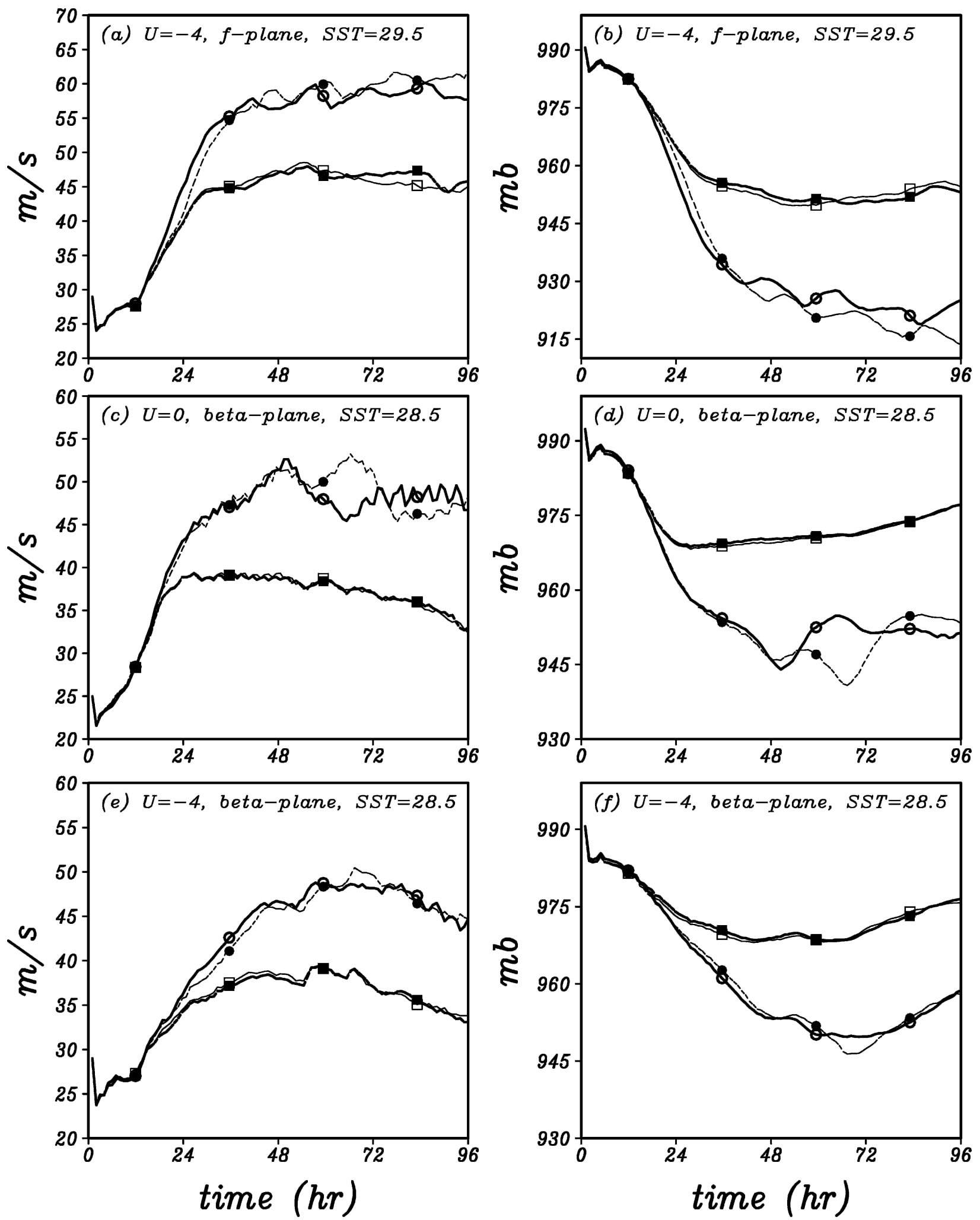

FIG. 3. Time series of the maximum wind speed $\left(\mathrm{m} \mathrm{s}^{-1}\right.$, left) and minimum central pressure (mb, right) in experiments E1 (a), (b); E2 (c), (d); and E3 (e), (f). The open and solid circles denote the fixed and asymmetric-only SST forcing experiments while the open and solid squares indicate the coupled and symmetric-only SST forcing experiments. 

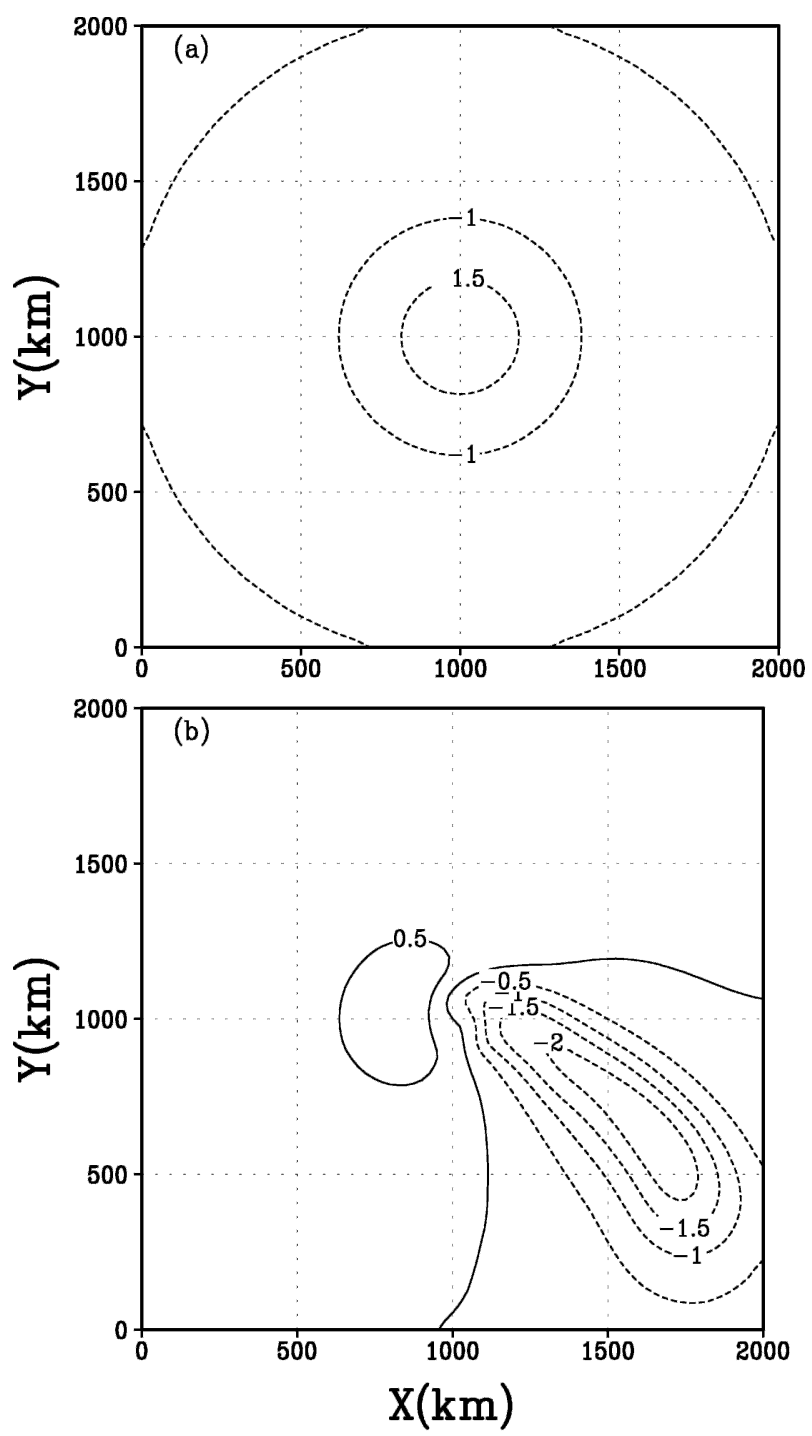

FIG. 4. Decomposition of the SST anomalies at $96 \mathrm{~h}$ resulting from hurricane-ocean interaction in the coupled experiment of set E2 (E2C): (a) the symmetric SST component and (b) the asymmetric SST component $\left({ }^{\circ} \mathrm{C}\right)$. Contour intervals are $0.5^{\circ} \mathrm{C}$.

maximum heating region. Willoughby (1992) investigated asymmetric convective forcing in a tropical cyclone as a mass source-sink pair. When the mass source sink maintains the same orientation with respect to the center, quasi-stationary gyres are established and persistently advect the vortex center toward the enhanced convection. In an example, the TC was shifted by $20 \mathrm{~km}$ in $18 \mathrm{~h}\left(0.3 \mathrm{~m} \mathrm{~s}^{-1}\right)$. Since the diabatic heating is dominated by the latent heat release, the asymmetry of diabatic heating can be displayed with the total rainfall rates. Figure 7 shows the time evolution of the rainfall rates for the fixed SST (E1F), the asymmetric-only SST forcing (E1A), and the symmetric-only SST forcing
(E1S) experiments in set E1. The mechanisms responsible for the generation of the rainfall asymmetry have been discussed in previous studies (Shapiro 1983; Peng et al. 1999; $\mathrm{Wu}$ and Braun 2004).

The rainfall asymmetries are changed in response to the time-dependent symmetric and asymmetric SST forcings. After $24 \mathrm{~h}$, the rainfall rate in the experiment with asymmetric-only SST forcing (Fig. 7b) increases compared with the fixed SST experiment (Fig. 7a). A possible reason is that the slightly positive SST anomalies ahead of the TC center enhance the surface fluxes and thus the rainfall. Second, the higher SST than that in the coupled and symmetric-only SST forcing experiments leads to a more intense TC circulation in the asymmetric-only SST forcing and fixed-SST experiments. Because the stronger storms axisymmetrize the asymmetries more effectively than the weaker storms, the asymmetries appear less in the fixed-SST and asymmetric forcing experiments. This is consistent with the fact that intense hurricanes tend to be rather symmetric (Willoughby et al. 1984).

Although the TCs are primarily steered by the easterly environmental flow, the track differences shown in Fig. 8 are consistent with the asymmetries of the rainfall pattern. Note that the ordinate scale is much smaller than the abscissa in Fig. 8. In the symmetric-only SST forcing experiment (Fig. 7c), the strongest rainfall is always on the northern side after $24 \mathrm{~h}$, and the TC persistently moves northwestward. In the fixed SST experiment (Fig. 7a), the TC persistently moves northwestward by $72 \mathrm{~h}$. The shift of the rainfall asymmetry from the northwest to the west since $60 \mathrm{~h}$ causes the TC to move westward. In the asymmetric-only SST forcing experiment (Fig. 7b), the region with enhanced rainfall shifts to the southwestern side, leading to a southward shift in the TC track after $48 \mathrm{~h}$.

For the fixed SST experiment in sets E2 and E3, the TCs move northwestward (Figs. 6b,c). As time progresses, the beta gyres develop and intensify, gradually leading to a shift in the precipitation pattern. In addition to the mechanism discussed for E1, the beta gyres and their associated southeasterly vertical shear can also affect the asymmetries of the precipitation pattern (Peng et al. 1999; Frank and Ritchie 1999, 2001). The rainfall in E2F at $24 \mathrm{~h}$ intensifies primarily ahead of the TC center (Fig. 9a). At 48 and $72 \mathrm{~h}$, the shift of the primary rainfall rate maximum to the eastern side results from the impacts of the beta gyres. For the fixedSST experiment of set E3 (Fig. 10a), the rainfall pattern is similar to that E1F shown in Fig. 7a, which suggests the dominant influence of the environmental flow. In the presence of air-sea coupling (Figs. 9b and 10b), the 
(a)

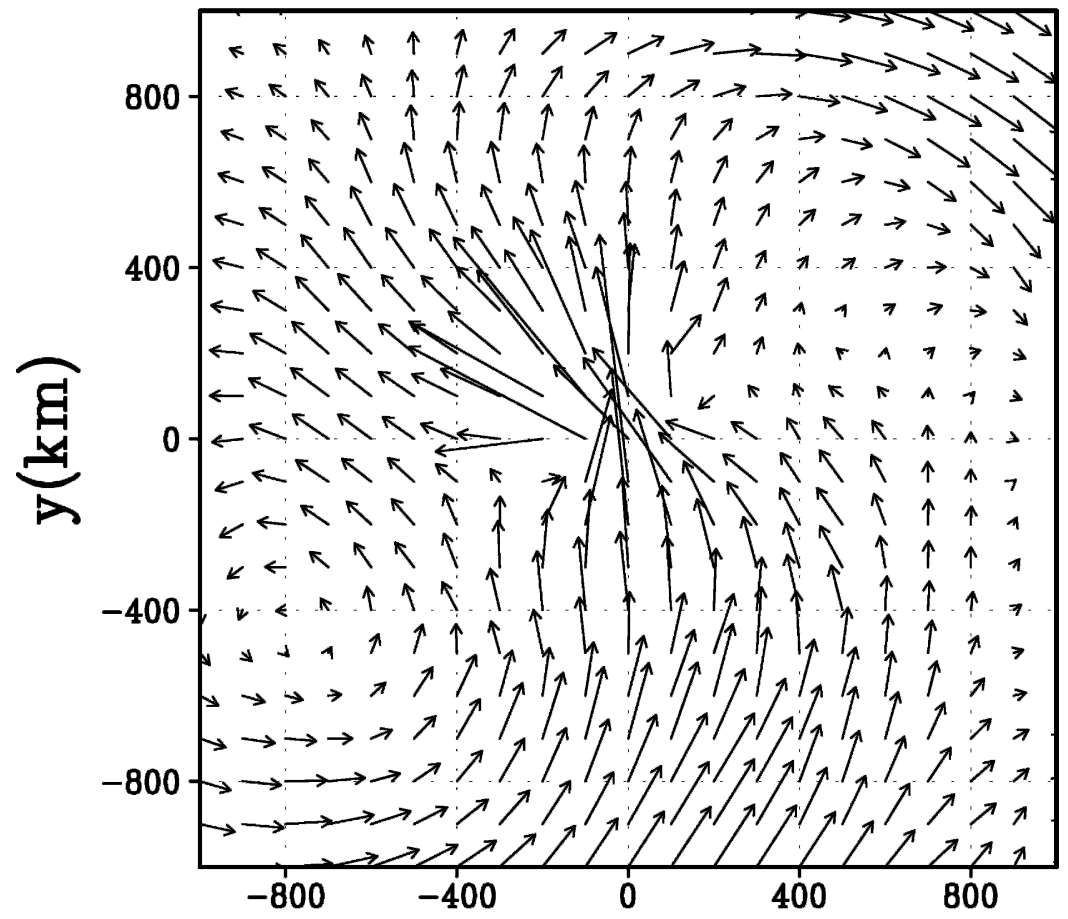

(b)

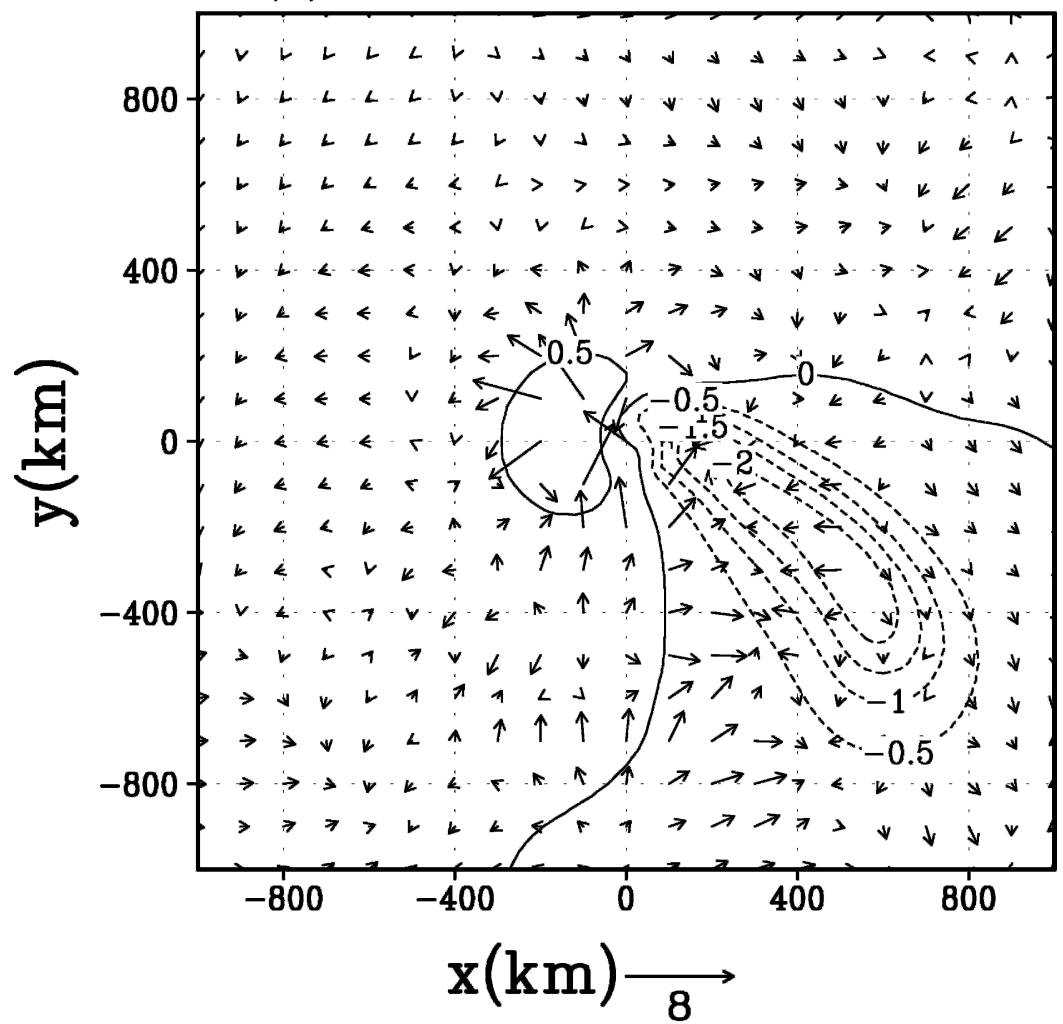

FIG. 5. (a) The lowest-level (about $990 \mathrm{mb}$ ) asymmetric wind field in the fixed SST experiment of set E2 (E2F) and (b) the wind difference between the asymmetric-only SST forcing (E2A) and fixed SST (E2F) experiments at $96 \mathrm{~h}$. The asymmetric component of the SST anomalies is superposed at an interval of $0.5^{\circ} \mathrm{C}$. The TC is located at the domain center. 

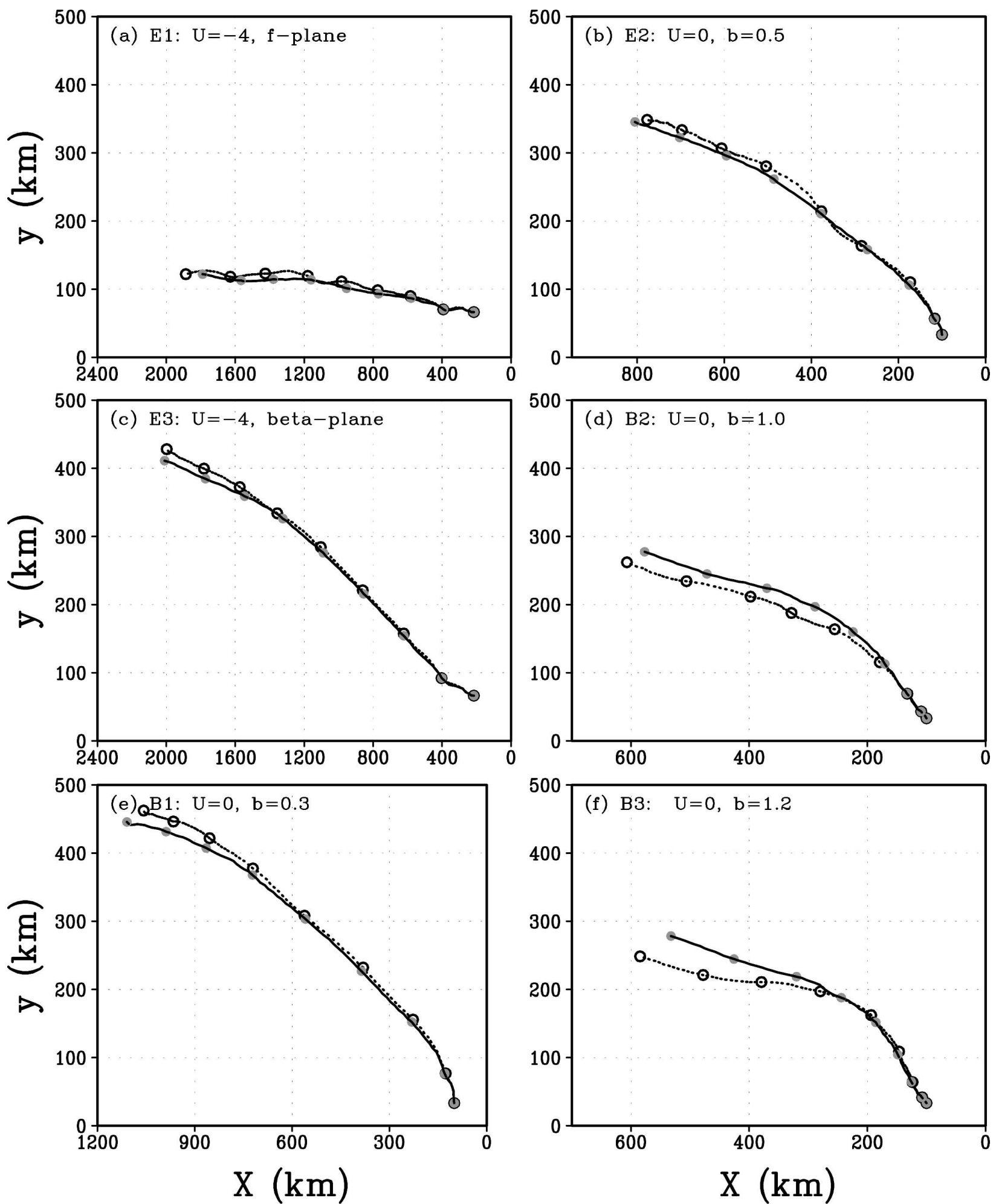

FIG. 6. The TC tracks in the fixed SST (dashed) and coupled (solid) experiments for sets (a) E1, (b) E2, (c) E3, (d) B2, (e) B1, and (f) B3. The TCs generally move westward and the center locations every $12 \mathrm{~h}$ are indicated with circles. 
(a)

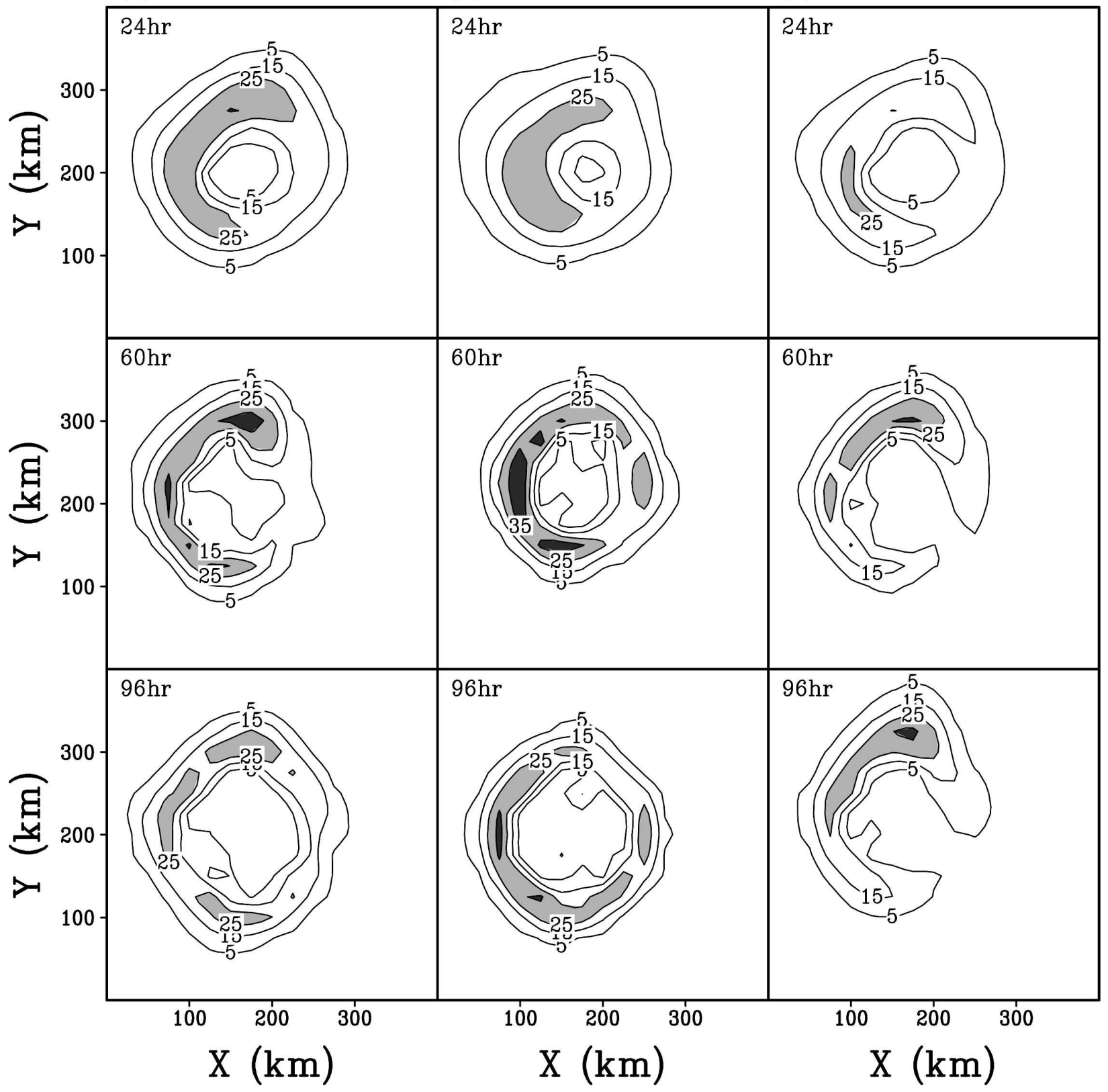

FIG. 7. Evolution of rainfall rates $\left(\mathrm{mm} \mathrm{h}^{-1}\right)$ in (a) the fixed SST, (b) asymmetric-only SST forcing, and (c) symmetric-only SST forcing experiments of set E1. The contour intervals are $5 \mathrm{~mm} \mathrm{~h}^{-1}$.

rainfall patterns exhibit more prominent wavenumber-1 asymmetries. With the maxima of the rainfall rates generally occurring in the southern region of the westward-propagating TCs after $24 \mathrm{~h}$, we hypothesize that the favorable location is associated with the asymmetric SST anomalies resulting from air-sea coupling. If we consider the cyclonic rotation of air parcels as they rise, the enhanced (suppressed) rainfall rates are related to the positive (negative) SST anomalies ahead of (behind) the TC center. These persistent rainfall asymmetries tend to shift the TC tracks slightly southward in the coupled experiments of sets E2 and E3 (Figs. 7b,c).

Bender et al. (1993) suggested that in experiments with air-sea interaction, a systematic weakening of the mean tangential flow at all radii alters the orientation of the beta gyres and thus affects the beta drift. The resulting secondary steering flow associated with the beta 


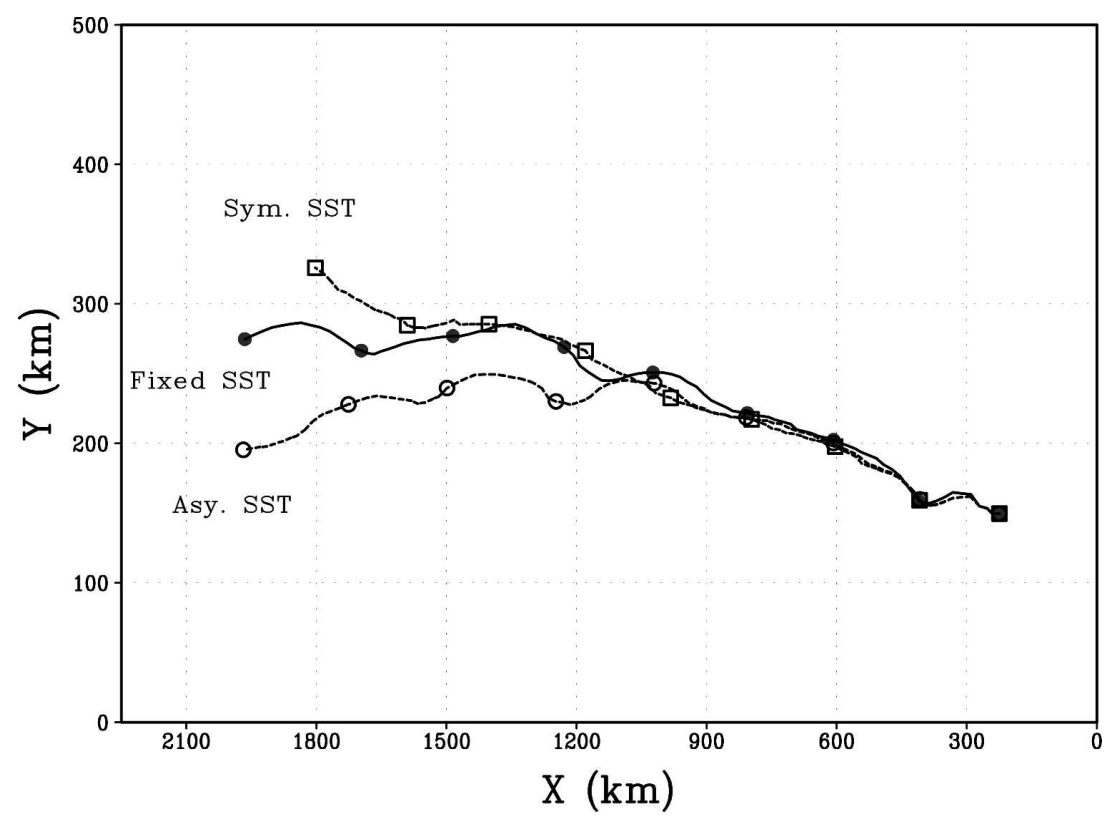

FIG. 8. The TC tracks in the fixed (solid circles), symmetric-only (open squares), and asymmetric-only (open circles) SST experiments for set E1. The TCs generally move westward and the center positions are indicated every $12 \mathrm{~h}$.

gyres in the coupled experiments rotates anticyclonically relative to that in the fixed-SST experiments (Fiorino and Elsberry 1989; Wang et al. 1997). As a result, the westward tracks gradually turn more to the north relative to the track in the fixed SST experiments. The influence of the beta drift and its association with the outer vortex strength can be investigated by adjusting the shape parameter $(b)$ of the initial vortex profile. In $\mathrm{E} 1, \mathrm{E} 2$, and $\mathrm{E} 3, b=0.5$. When $b$ is made smaller (larger) than 0.5 , the outer portion of the vortex is strengthened (weakened). Since the resulting SST cooling is primarily a function of TC intensity and translation, the weakening of the TC circulation is nearly independent of the outer vortex strength or the shape parameter. In this case, the relative decrease in the outer vortex strength is larger for a vortex with initially weak strength than for one with initially strong strength (Fig. 11). Thus the change in the northward beta drift is larger for the former than for the latter. While the influence of the asymmetric diabatic heating remains the same, in agreement with Khain and Ginis (1991) the vortex track in the coupled model shifts more southward when the outer part of the vortex is stronger $(b=$ 0.3; Fig. 6e), and in agreement with Bender et al. (1993) the vortex in the coupled model shifts more northward when the vortex strength is weaker $(b=1.0$ and 1.2; Figs. 6d,f).

Therefore the influence of air-sea interaction on TC tracks is a result of the competing effects of the asym- metries associated with the resulting SST anomalies and changes in the beta drift associated with the outer wind weakened by the air-sea coupling. For this reason, the net track differences between the coupled and fixed-SST experiments are generally small.

\section{Conclusions and discussion}

The impacts of air-sea interaction on TC intensity and track are investigated using a coupled hurricaneocean model. Three sets of numerical experiments are designed with idealized environmental influences. For each set, four experiments are conducted with fixed SST, air-sea coupling, symmetric-only SST forcing, and asymmetric-only SST forcing, respectively. In the latter two experiments, the time-dependent SST forcing is deduced from the hourly output of the corresponding coupled experiments. The coupled model, which consists of a hydrostatic hurricane model and an intermediate ocean model with an OML and a middle thermocline layer. Compared with observations and previous numerical simulations, can reasonably produce the major features of the ocean responses to moving TC forcing, including OML deepening, SST cooling, and OML and thermocline layer currents.

Although the negative SST anomalies associated with the asymmetric component are generally larger in magnitude than those associated with the symmetric component, the influence of the asymmetric compo- 
(a)

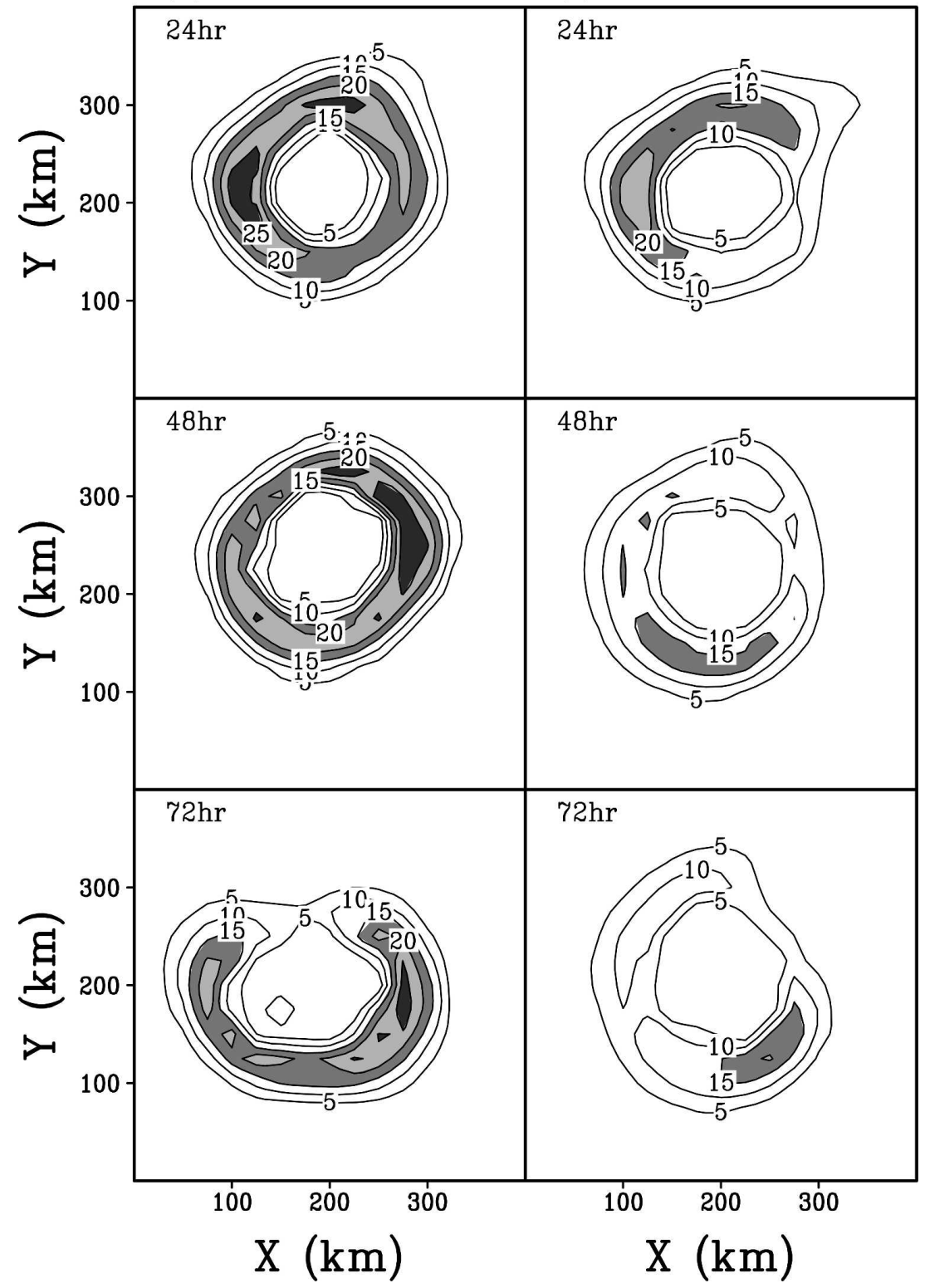

FIG. 9. Evolution of rainfall rates $\left(\mathrm{mm} \mathrm{h}^{-1}\right)$ in the (a) fixed SST and (b) coupled experiments of set E2.

nent is insignificant while the resulting symmetric cooling plays a decisive role in weakening TCs in the coupled experiments. The asymmetric SST forcing affects TC intensity only through the resulting asymmetries in the TC circulation, but the asymmetric winds induced by the asymmetric SST forcing are mainly confined to the lower boundary and are much weaker than the asymmetric winds induced by large-scale environmental influences such as uniform flows and the beta effect. This result concurs with Emanuel's (1999) finding that the evolution of storm intensity can be success- fully simulated by including only the symmetric SST decreases caused by air-sea coupling.

The asymmetric SST forcing intensifies the rainfall rates on the front left-hand side (relative to TC motion). The symmetric SST forcing weakens the TC circulation and thus the axisymmetrization process, thus reinforcing convective asymmetry, meanwhile, the weakening of the outer portion of the mean vortex alters the orientation of the beta gyres and leads to more northward beta drift. For the experiments including the beta effect (E2 and E3), the enhanced TC heating 
(a)

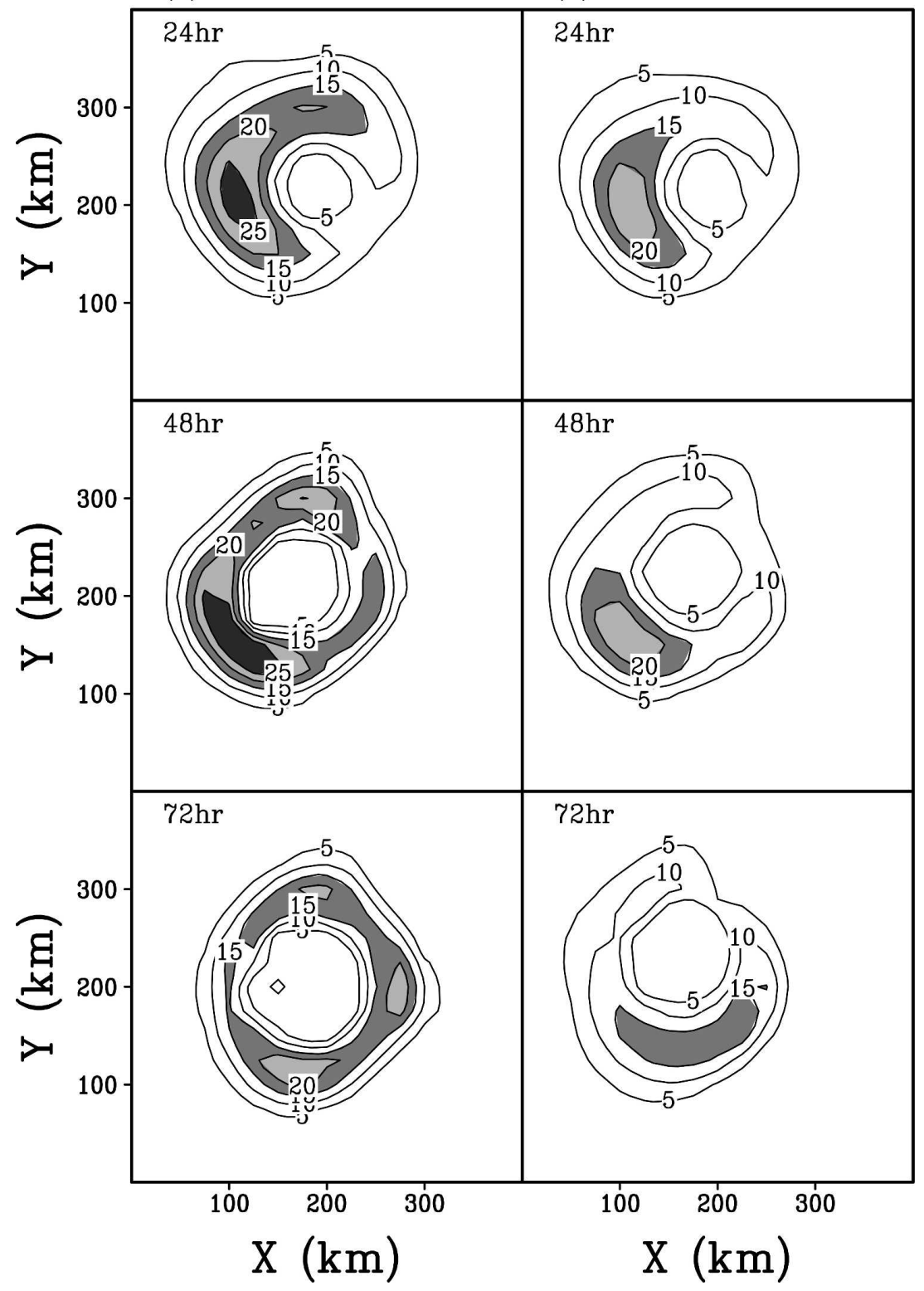

FIG. 10. Same as Fig. 9 but for E3.

asymmetries tend to shift westward-moving TCs southward while the modified beta drift tends to shift TCs northward in the coupled experiments. Because of the opposing effects, the resulting track difference between the fixed-SST and coupled experiments is generally small. The different effects of air-sea interaction on TC tracks simulated by Khain and Ginis (1991) and Bender et al. (1993) can be reconciled by adjusting the vortex outer strength. When the outer vortex strength is relatively weak (strong), a TC in the coupled model moves to the north (south) of the TC in the corresponding fixed SST experiments.
We should mention that this study particularly focuses on the different ways the symmetric and asymmetric components of the SST anomalies induced by air-sea interaction affect TC intensity and track. Compared with the complicated hurricane-ocean coupling in real cases, the complexity of the coupled model used in this study has been largely simplified. Although the model can simulate the most important features we know so far, some caveats shall be kept in mind as we interpret these results. First, due to the relatively coarse horizontal model resolution, there is no explicit convection schemes for the complex moist processes that are 


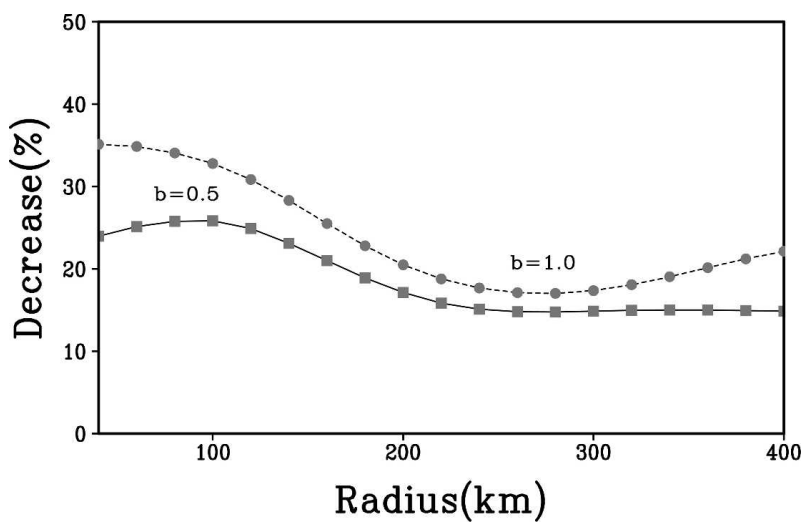

FIG. 11. Relative decreases of the $850-\mathrm{mb}$ symmetric tangential wind at $72 \mathrm{~h}$ due to air-sea interaction for cases $\mathrm{b}=0.5(\mathrm{E} 2 \mathrm{C}$, solid) and $\mathrm{b}=1.0(\mathrm{~B} 2$, dashed).

very important for TCs. Second, the boundary layer is parameterized with the simple bulk scheme, and some important processes in the inflow boundary layer such as sea spray are not included in this coupled model (Barnes et al. 2004). These caveats may make the extents and magnitudes of the simulated ocean cooling and the atmospheric response such as precipitation less realistic. However, we believe that the simplification in the model physics may not qualitatively affect the results presented in this paper.

Acknowledgments. The authors thank three anonymous reviewers for their thorough editorial efforts and insightful comments on an earlier version of the paper, which lead to much improvement in the presentation of this study. Liguang Wu and Scott Braun thank Dr. R. Kadar (NASA HQ) for his support through the NASA CAMEX program. Bin Wang acknowledges the support by Office of Naval Research under Award N00014-021-0532.

\section{REFERENCES}

Barnes, G., R. Schneider, and S. Houston, 2004: Vertical profiles of thermodynamic variables in Hurricanes Bonnie (1998) and Mitch (1998): Implications for energy transport into the inflow layer. Preprints, 26th Conf. on Hurricanes and Tropical Meteorology, Miami, FL, Amer. Meteor. Soc., 653-654.

Bender, M. A., and I. Ginis, 2000: Real-case simulations of hurricane-ocean interaction using a high resolution coupled model: Effects on hurricane intensity. Mon. Wea. Rev., 128, 917-946.

- - - and Y. Kurihara, 1993: Numerical simulations of hurricane-ocean interaction with a high resolution coupled model. J. Geophys. Res., 98, 23 245-23 263.

Black, P. G., 1983: Ocean temperature changes induced by tropical cyclones. Ph.D. dissertation, Pennsylvania State University, $278 \mathrm{pp}$.

Brink, K. H., 1989: Observations of the response of the ther- mocline currents to a hurricane. J. Phys. Oceanogr., 19, 10171022.

Chan, J. C.-L., Y. Duan, and L. K. Shay, 2001: Tropical cyclone intensity changes from a simple ocean-atmosphere coupled model. J. Atmos. Sci., 58, 154-172.

Chang, S. W., and R. A. Anthes, 1979: The mutual response of the tropical cyclone and the ocean. J. Phys. Oceanogr., 9, 128135

Deardorff, J. W., 1983: A multi-limit layer entrainment formulation. J. Phys. Oceanogr., 13, 988-1002.

Elsberry, R. L., T. S. Fraim, and R. N. Trapnell Jr., 1976: Mixed layer model of the oceanic thermal response to hurricanes. $J$. Geophys. Res., 81, 1153-1163.

Emanuel, K. A., 1986: An air-sea interaction theory for tropical cyclones. Part I: Steady-state maintenance. J. Atmos. Sci., 43, 585-604.

_ 1999: Thermodynamic control of hurricane intensity. $\mathrm{Na}$ ture, 401, 665-669.

Falkovich, A. I., A. P. Khain, and I. Ginis, 1995: Motion and evolution of binary tropical cyclones in a coupled atmosphereocean numerical model. Mon. Wea. Rev., 123, 1345-1363.

Fernando, H. J., 1991: Turbulent mixing in stratified fluids. Annu. Rev. Fluid Mech., 23, 455-493.

Fiorino, M. J., and R. L. Elsberry, 1989: Some aspects of vortex structure related to tropical cyclone motion. J. Atmos. Sci., 46, 975-990.

Frank, W. M., and E. A. Ritchie, 1999: Effects on environmental flow upon tropical cyclone structure. Mon. Wea. Rev., 127, 2044-2061.

— and - 2001: Effects of vertical wind shear on the intensity and structure of numerically simulated hurricanes. Mon. Wea. Rev., 129, 2249-2269.

Geisler, J. E., 1970: Linear theory of the response of a two layer ocean to a moving hurricane. Geophys. Fluid Dyn., 1, 249272

Gill, A. E., 1984: On the behavior of inertial waves in the wakes of storm. J. Phys. Oceanogr., 14, 1129-1151.

Ginis, I., 1995: Ocean response to tropical cyclones. Global perspectives on tropical cyclones. WMO/TD 693, 218-256.

Khain, A. P., and I. Ginis, 1991: The mutual response of a moving tropical cyclone and the ocean. Beitr. Phys. Atmos., 64, 125141.

Kondo, J., 1975: Air-sea bulk transfer coefficients in diabatic conditions. Bound.-Layer Meteor., 9, 91-112.

Kraus, E. B., and J. S. Turner, 1967: A one-dimensional model of the seasonal thermocline. Part II: The general theory and its consequences. Tellus, 19, 98-105.

Kuo, H. L., 1974: Further studies of the parameterization of the influence of cumulus convection on large-scale flow. J. Atmos. Sci., 31, 1232-1240.

Leslie, L. M., G. A. Mills, L. W. Logan, D. J. Gauntlett, G. A. Kelly, M. J. Manton, J. L. McGregor, and J. M. Sardie, 1985: A high resolution primitive equations NWP model for operations and research. Aust. Meteor. Mag., 33, 11-35.

Louis, J. F., 1979: A parametric model of vertical eddy fluxes in the atmosphere. Bound.-Layer Meteor., 17, 746-756.

Peng, M. S., B.-F. Jeng, and R. T. Williams, 1999: A numerical study on tropical cyclone intensification. Part I: Beta effect and mean flow effect. J. Atmos. Sci., 56, 1404-1423.

Price, J. F., 1981: Upper ocean response to a hurricane. J. Phys. Oceanogr., 11, 153-175.

Rotunno, R., and K. A. Emanuel, 1987: An air-sea interaction theory for tropical cyclones. Part II: Evolutionary study using 
a nonhydrostatic axisymmetric numerical model. J. Atmos. Sci., 44, 542-561.

Sanford, T. B., P. G. Black, J. R. Haustein, J. W. Feeney, G. Z. Forristall, and J. F. Price, 1987: Ocean response to a hurricane. Part I: Observations. J. Phys. Oceanogr., 17, 2065-2083.

Shapiro, L. J., 1983: Asymmetric boundary layer flow under a translating hurricane. J. Atmos. Sci., 40, 1984-1998.

Shay, L. K., and R. L. Elsberry, 1987: Near-inertial ocean current response to Hurricane Frederic. J. Phys. Oceanogr., 17, 12491269.

, P. G. Black, A. J. Mariano, J. D. Hawkins, and R. L. Elsberry, 1992: Upper ocean response to Hurricane Gilbert. $J$. Geophys. Res., 97, 20 227-20 248.

Sutyrin, G. G., and A. P. Khain, 1979: Interaction of the ocean and the atmosphere in the area of moving tropical cyclones. Dokl. Akad. Sci. USSR, 249, 467-470.

Tuleya, R. E., and Y. Kurihara, 1982: A note on the sea surface temperature sensitivity of a numerical model of tropical storm genesis. Mon. Wea. Rev., 110, 2063-2069.

Wang, B., T. Li, and P. Chang, 1995: An intermediate model of the tropical Pacific Ocean. J. Phys. Oceanogr., 25, 1599-1616.

- X. Li, and L. Wu, 1997: Hurricane beta drift direction in horizontally sheared flows. J. Atmos. Sci., 54, 1462-1471.
Wang, Y., 1998: On the bogusing of tropical cyclones in numerical models: The influence of vertical structure. Meteor. Atmos. Phys., 65, 153-170.

Willoughby, H. E., 1992: Linear motion of a shallow-water barotropic vortex as an initial-value problem. J. Atmos. Sci., 49, 2015-2031.

—, F. D. Marks Jr., and R. J. Feinberg, 1984: Stationary and moving convective bands in hurricanes. J. Atmos. Sci., 41, 3189-3211.

Wu, L., 1999: Study of tropical cyclone motion with a coupled hurricane-ocean model. Ph.D. dissertation, University of Hawaii at Manoa, $212 \mathrm{pp}$.

_ and B. Wang, 2000: A potential vorticity tendency diagnostic approach for tropical cyclone motion. Mon. Wea. Rev., 128, 1899-1911. , and _ 2001: Effects of convective heating on movement and vertical coupling of tropical cyclones: A numerical study. J. Atmos. Sci., 58, 3639-3649.

— asymmetries on hurricane intensity: A numerical study. $J$. Atmos. Sci., 61, 3065-3081. 